\title{
Effects of grape seed extract on dyslipidaemia: a systematic review and dose-response meta-analysis of randomised controlled trials
}

\author{
Javad Anjom-Shoae ${ }^{1,2}$, Alireza Milajerdi ${ }^{1,2}$, Bagher Larijani ${ }^{3}$ and Ahmad Esmaillzadeh ${ }^{2,4,5 *}$ \\ ${ }^{1}$ Students' Scientific Research Center, Tehran University of Medical Sciences, PO Box 1417755331, Tehran, Iran \\ ${ }^{2}$ Department of Community Nutrition, School of Nutritional Sciences and Dietetics, Tehran University of Medical Sciences, \\ Tehran, Iran \\ ${ }^{3}$ Endocrinology and Metabolism Research Center, Endocrinology and Metabolism Clinical Sciences Institute, Tehran University \\ of Medical Sciences, Tehran, Iran \\ ${ }^{4}$ Obesity and Eating Habits Research Center, Endocrinology and Metabolism Molecular-Cellular Sciences Institute, Tehran \\ University of Medical Sciences, PO Box 1414413137, Tehran, Iran \\ ${ }^{5}$ Food Security Research Center, Department of Community Nutrition, School of Nutrition and Food Science, Isfahan University \\ of Medical Sciences, PO Box 8174673461 , Isfahan, Iran
}

(Submitted 14 May 2019 - Final revision received 28 February 2020 - Accepted 28 February 2020 - First published online 6 March 2020)

Abstract

Data on the effect of grape seed extract (GSE) on lipid profiles are inconclusive. We undertook a systematic review and meta-analysis of randomised controlled clinical trials on the effect of GSE on serum lipid profiles. The online databases of PubMed, ISI Web of Science, Scopus, ProQuest, Science Direct and Embase were searched for relevant publications until March 2019, using MeSH and non-MeSH keywords. Study selection, data extraction and quality assessment were completed independently by two investigators. Meta-regression and subgroup analyses were performed to identify the source of heterogeneity. Assessment of study quality was conducted using the Jadad scale. Eleven randomised clinical trials involving 536 participants were included in the present meta-analysis. Combining effect sizes from earlier studies, we found that GSE supplementation significantly decreased serum levels of LDL-cholesterol ( $-0 \cdot 17 \mathrm{mmol} / \mathrm{l} ; 95 \% \mathrm{CI}-0 \cdot 34,-0 \cdot 01)$ and TAG $(-0 \cdot 11 \mathrm{mmol} / \mathrm{l} ; 95 \% \mathrm{CI}-0 \cdot 18,-0 \cdot 05)$. Although no overall significant effect of GSE supplementation on circulating total- and HDL-cholesterol levels was observed, there were significant reductions in these lipids in studies with $<10$ weeks of intervention and those that had administered the dosages of $<300 \mathrm{mg} / \mathrm{d}$ of GSE. In conclusion, GSE supplementation seems to favourably affect serum levels of LDL and TAG concentrations, but it did not affect total- and HDL-cholesterol concentrations.

Key words: Grape seed extract: Lipid profiles: Meta-analyses: Randomised controlled trials

Flavonoids, as a major component of grape and grape products, have been reported to have several favourable effects on human health $^{(1)}$. Grape seed extracts (GSE) contain a high concentration of flavonoids, in particular proanthocyanidins, potent antioxidants with many cardiovascular benefits ${ }^{(2,3)}$. Proanthocyanidins are also known as condensed tannins existing in GSE as monomeric (catechin and epicatechin), dimeric, trimeric and polymeric tannin structures $^{(4)}$. Findings from in vitro studies have demonstrated that these compounds act as free-radical scavengers and may prevent the oxidation of LDL-cholesterol ${ }^{(5-7)}$; therefore, they have an important role in decreasing the progression of $\mathrm{CVD}^{(8)}$. Their free radical-scavenging ability has been indicated to be even fifty times greater than that of vitamins $\mathrm{C}$ and $\mathrm{E}$ and $\beta$-carotene ${ }^{(9,10)}$.
A number of these studies have also shown positive effects of flavonoids upon novel vascular risk factors such as inflammation ${ }^{(11)}$. However, in vivo experience has provided less clear results ${ }^{(12-17)}$. Although a recent investigation has shown that GSE significantly reduced plasma cholesterol in rats fed a high-fat diet ${ }^{(12)}$, few clinical trials have explored this issue in humans, some with promising results. For instance, in an Italian study, conducted on twenty-four heavy smokers, no significant change in plasma lipid profiles was found after 4 weeks supplementation with $75 \mathrm{mg}$ GSE twice daily; however, GSE resulted in a decreased susceptibility of LDL-cholesterol to oxidation ${ }^{(13)}$. Conversely, combined administration of niacin-bound $\mathrm{Cr}$ and GSE decreased total cholesterol (TC) and LDL-cholesterol after 2 months among forty

Abbreviations: GSE, grape seed extract; TC, total cholesterol.

* Corresponding author: Ahmad Esmaillzadeh, fax +98-21-88984861, email a-esmaillzadeh@sina.tums.ac.ir 
hypercholesterolaemic individuals ${ }^{(14)}$. In addition, administration of GSE for 8 weeks in mild hyperlipidaemic patients resulted in improved lipid profiles ${ }^{(15)}$. This was also reported in diabetic patients ${ }^{(16)}$. Nonetheless, Hansen et al. reported no significant effect of GSE on serum lipids in sixty-nine healthy individuals ${ }^{(17)}$. The origin, dosage and composition of polyphenolic extracts from the grape as well as different study designs along with the health conditions of the study participants might provide some reasons for these discrepancies.

Despite several publications on the effects of GSE on serum lipid profiles, we are aware of no study summarising earlier publications in this regard. Given the controversial findings in previous publications, this study was done to systematically review earlier publications on the impact of GSE administration on lipid profiles and to perform a meta-analysis of relevant randomised controlled trials in this regard.

\section{Methods}

This study was performed based on the guidelines of the Preferred Reporting Items for Systematic Reviews and MetaAnalysis (PRISMA) statement ${ }^{(18)}$.

\section{Search strategy}

A systematic search was carried out in the online databases of PubMed, ISI Web of Science, Scopus, ProQuest, Science Direct and Embase for relevant publications until March 2019. The keywords used in our search strategy were ('Grape seed extract' [Mesh] OR 'Polyphenols'[Mesh] OR 'Proanthocyanidins' [Mesh] OR 'Vitis' [Mesh] OR 'Grape seed' [tiab] OR 'Polyphenols' [tiab] OR 'Proanthocyanidins' [tiab] OR 'Vitis' [tiab]) AND ('Lipids' [Mesh] OR 'Cholesterol' [Mesh] OR 'Cholesterol, VLDL' [Mesh] OR 'Cholesterol, HDL' [Mesh] OR 'Lipoproteins, HDL' [Mesh] OR 'High-Density Lipoproteins, Pre-beta' [Mesh] OR 'Cholesterol, LDL' [Mesh] OR 'Triglycerides' [Mesh] OR 'Lipoproteins' [Mesh] OR 'Hypercholesterolemia' [Mesh] OR 'Hyperlipidemias' [Mesh] OR 'Dyslipidemias' [Mesh] OR 'lipids' [tiab] OR 'cholesterol' [tiab] OR 'triglyceride*' [tiab] OR 'triacylglycerol' [tiab] OR 'HDL' [tiab] OR 'LDL' [tiab] OR 'Hypercholesterolemia' [tiab] OR 'Hyperlipidemias' [tiab] OR 'Dyslipidemias' [tiab]) AND ('Clinical Trial' OR 'trial' OR 'intervention'). We considered no restriction on time of publication and language. In addition, the reference lists of the relevant papers were also hand-searched to identify further relevant studies. In the search strategy, unpublished studies were excluded.

\section{Inclusion criteria}

We included the studies in the present meta-analysis if they met the following criteria: (1) studies that investigated the effect of GSE on any of the lipid profile parameters, including TC, HDL-cholesterol, LDL-cholesterol and TAG; (2) those that were of randomised controlled clinical trial; (3) those that presented sufficient information on plasma/serum lipid levels at study baseline and at the end of trial and (4) those trials that were done on healthy participants or individuals only with chronic diseases including dyslipidaemia, diabetes mellitus, the metabolic syndrome and breast cancer. In the case of duplicate publications from the same study group, a study with the larger sample size or more complete information was retained.

\section{Data extraction}

According to the predefined inclusion criteria, two investigators independently completed the search, data extraction and quality assessment, and any discrepancies between the two reviewers were resolved through discussion. Data of interest from each individual study were extracted as follows: first author, year of publication, country of origin, study design, the duration of the intervention, sample size, sex, mean age and BMI at study baseline, the type and dose of GSE supplementation, participants' baseline health status and the means, and standard deviations of serum concentrations of lipids at study baseline and post-intervention. The data for blood lipids were converted into the same units $(\mathrm{mmol} / \mathrm{l})$, and mean differences in concentrations of plasma lipids (TC, LDL-cholesterol, HDL-cholesterol and TAG) between the control and GSE groups were calculated. If a study had reported the effect sizes for two different doses of GSE (low- and high-dose), each arm was considered as a separate study.

\section{Excluded studies}

In this meta-analysis, letters, comments, short communications, reviews, ecological studies and animal studies were excluded from the analysis. In our initial search, we found 531 articles. On the basis of title and abstract, we excluded 512 studies and nineteen remaining articles were reviewed in full text. Another eight papers were further excluded because of the following reasons: (1) studies that investigated the effect of whole grape or grape juice supplementation on lipid profiles ( $n 3$ ); (2) those that evaluated the administration of grape powder $(n 2)$ and (3) publications that assessed the impact of red wine supplementation on lipid profiles ( $n$ 3). Finally, eleven randomised clinical trials that met our inclusion criteria were included in this analysis (Fig. 1).

\section{Assessment of study quality}

Study quality was assessed by using Jadad scale ${ }^{(19)}$, in which the total score ranges from 0 to 5 points based on the following criteria: (1) randomisation; (2) appropriate method for randomisation; (3) double-blinding; (4) appropriate method for doubleblinding and (5) description of dropouts and withdrawals. In the present study, trials scored one point for each area addressed in the study design, with a possible score of 0 to 5 (highest level of quality). Any discrepancies were resolved by discussion. We defined high-quality publications as those that had the Jadad score of 3 or more (Table 1 ).

\section{Statistical analysis}

The overall effect sizes were calculated using mean differences and standard deviations of plasma lipids (TC, LDL-cholesterol, HDL-cholesterol and TAG). In studies that had reported standard errors, we computed SD using CI or $P$ values based on the standard formula ${ }^{(20,21)}$. If the SD of the mean difference 

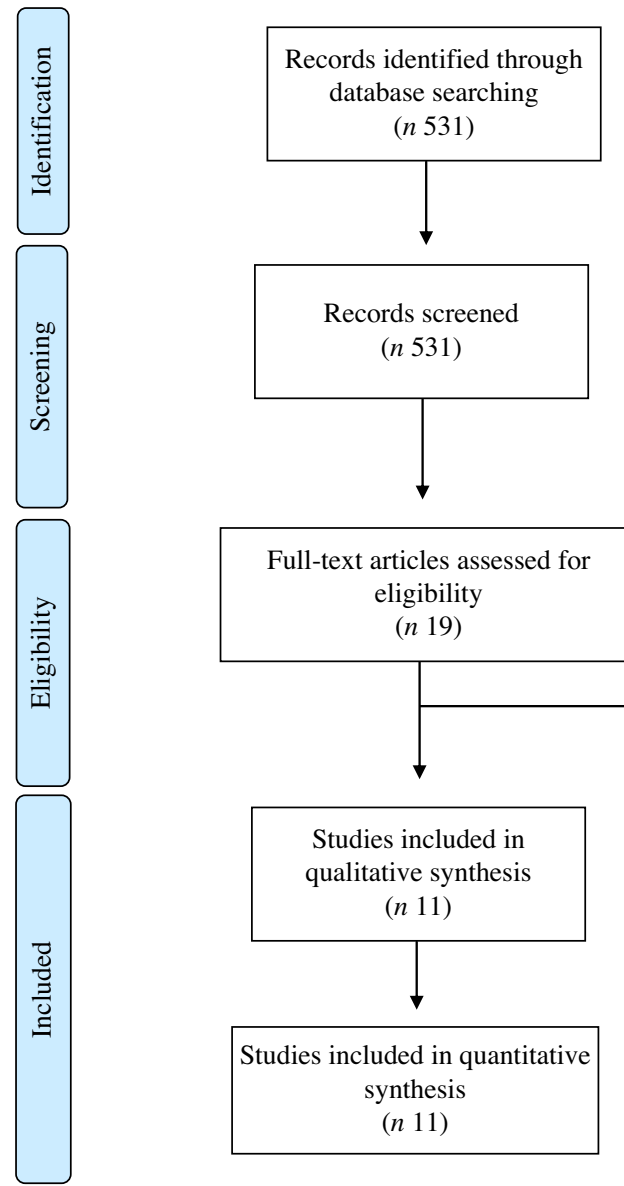

Fig. 1. Flow diagram of study selection.

was not stated in the publication, we calculated it using the following formula: $\mathrm{SD}_{\text {change }}=\sqrt{\left(\mathrm{SD}_{\text {baseline }}\right) 2}+\sqrt{\left(\mathrm{SD}_{\text {final }}\right) 2}$ $-\sqrt{2 \times 0.9 \times \mathrm{SD}_{\text {baseline }} \times \mathrm{SD}_{\text {final }}}(20)$. Then, the meta-analysis was conducted to calculate the overall weighted mean difference pooling study-specific mean difference through random effects model. We also applied meta-regression to examine the effective dosage of GSE supplementation. To test dose-response relations, we plotted the relation between GSE dosage $(\mathrm{mg} / \mathrm{d})$ and the absolute mean change in each outcome using fractional polynomial models with the best-fitting model considered the one with the lowest deviance ${ }^{(22)}$. We applied Cochran's $Q$ test and $I^{2}$ to assess between-study heterogeneity ${ }^{(23)}$. To detect probable sources of heterogeneity, we did subgroup analysis. The predefined categories for subgroup analysis were as follows: geographical region (USA/non-USA countries), study design (parallel/crossover), duration of intervention ( $<10$ weeks $\geq 10$ weeks), sample size ( $<50 / \geq 50$ participants), sex (male/female/both sex), mean age at study baseline $\left(<50 / \geq 50\right.$ years), mean BMI at study baseline $\left(<27 / \geq 27 \mathrm{~kg} / \mathrm{m}^{2}\right)$, GSE dosage ( $<300 / \geq 300 \mathrm{mg} / \mathrm{d}$ ), supplementation type (capsule/ tablet/functional foods), participants' baseline status (healthy/ chronic condition) and dyslipidaemia (yes/no). In addition to main analyses, we conducted sensitivity analysis to find if the overall estimate depended on the effect size from a single study. Assessing the publication bias was done by visual inspection of funnel plots along with Egger's test. All statistical analyses were done using Stata software, version 11.2 (StataCorp). $P<0.05$ was considered as statistically significant.

\section{Results}

Among 531 retrieved publications, eleven papers met the inclusion criteria and were selected for the present metaanalysis ${ }^{(13-16,24-30)}$. Two studies had two arms with two different doses ${ }^{(27,29)}$; therefore, we had thirteen effect sizes for the analysis.

\section{Findings from systematic review}

Characteristics of studies included in this systematic review are briefly described in Table 1 . These clinical trials were published between 2000 and 2016 and had recruited 536 participants in total, with individual study sizes ranging from 19 to 96 . Three publications were from the USA ${ }^{(14,25,29)}$, two studies from the $\mathrm{UK}^{(16,30)}$, two from $\operatorname{Iran}^{(15,28)}$ and one study from Australia, Spain, Italy and Japan ${ }^{(13,24,26,27)}$. All studies, except for one ${ }^{(27)}$, were double-blind controlled trials. Of eleven clinical trials, four had a crossover design ${ }^{(13,15,16,24)}$ and seven had a parallel $\operatorname{design}^{(14,25-30)}$. The duration of intervention varied from 4 to 
NS British Journal of Nutrition

Table 1. Baseline characteristics of all included clinical trials investigating impacts of grape seed extract (GSE) supplementation on plasma lipids

\begin{tabular}{|c|c|c|c|c|c|c|c|c|c|c|c|}
\hline Authors & Country & Design & $\begin{array}{l}\text { Sample size } \\
\quad \text { (total/ } \\
\text { intervention) }\end{array}$ & $\begin{array}{l}\text { Mean age of } \\
\text { intervention } \\
\text { (years) }\end{array}$ & $\begin{array}{l}\text { Mean BMI of } \\
\text { intervention } \\
\left(\mathrm{kg} / \mathrm{m}^{2}\right)\end{array}$ & $\begin{array}{l}\text { GSE dosage } \\
(\mathrm{mg} / \mathrm{d})\end{array}$ & $\begin{array}{l}\text { GSE } \\
\text { supplementation } \\
\text { type }\end{array}$ & $\begin{array}{l}\text { Duration of } \\
\text { follow-up } \\
\text { (weeks) }\end{array}$ & $\begin{array}{l}\text { Mean basal/final of } \\
\text { lipid profiles of } \\
\text { control (mmol/l) }\end{array}$ & $\begin{array}{l}\text { Mean basal/final } \\
\text { lipid profiles of } \\
\text { intervention (mmol/l) }\end{array}$ & $\begin{array}{l}\text { Jadad } \\
\text { score }\end{array}$ \\
\hline Preuss et al. ${ }^{(14)}$ & USA & $\begin{array}{l}\text { Double-blind/ } \\
\text { parallel }\end{array}$ & $19 / 10$ & NR & NR & 100 & Tablet & 8 & $\begin{array}{l}\text { TC: } 6 \cdot 49 / 6 \cdot 23 \\
\text { LDL: } 4 \cdot 13 / 4 \cdot 0 \\
\text { HDL: } 1 \cdot 42 / 1 \cdot 45 \\
\text { TAG: - }\end{array}$ & $\begin{array}{l}\text { TC: } 6 \cdot 33 / 6 \cdot 15 \\
\text { LDL: } 4 \cdot 13 / 4 \cdot 06 \\
\text { HDL: } 1 \cdot 24 / 1 \cdot 23 \\
\text { TAG: - }\end{array}$ & 4 \\
\hline Vigna et al. ${ }^{(13)}$ & Italy & $\begin{array}{l}\text { Double-blind/ } \\
\text { crossover }\end{array}$ & $48 / 24$ & 54 & 27 & 300 & Capsule & 14 & $\begin{array}{l}\text { TC: } 6 \cdot 09 / 6 \cdot 06 \\
\text { LDL: } 4 \cdot 15 / 4 \cdot 15 \\
\text { HDL: } 1 \cdot 09 / 1.09 \\
\text { TAG: } 1.91 / 1 \cdot 81\end{array}$ & $\begin{array}{l}\text { TC: } 5 \cdot 9 / 6 \cdot 09 \\
\text { LDL: } 4 \cdot 04 / 4 \cdot 15 \\
\text { HDL: } 1 \cdot 11 / 1 \cdot 11 \\
\text { TAG: } 1 \cdot 66 / 1 \cdot 84\end{array}$ & 3 \\
\hline Clifton $^{(24)}$ & Australia & $\begin{array}{l}\text { Double-blind/ } \\
\text { crossover }\end{array}$ & $70 / 35$ & 58 & 28.4 & 2000 & Functional food & 12 & $\begin{array}{l}\text { TC: } 6 \cdot 57 / 6 \cdot 64 \\
\text { LDL: } 4 \cdot 59 / 4 \cdot 63 \\
\text { HDL: } 1 \cdot 18 / 1 \cdot 15 \\
\text { TAG: } 1.80 / 1.92\end{array}$ & $\begin{array}{l}\text { TC: } 6 \cdot 57 / 6 \cdot 63 \\
\text { LDL: } 4 \cdot 59 / 4 \cdot 61 \\
\text { HDL: } 1 \cdot 18 / 1 \cdot 18 \\
\text { TAG: } 1 \cdot 80 / 1 \cdot 88\end{array}$ & 3 \\
\hline Brooker et al. ${ }^{(30)}$ & UK & $\begin{array}{l}\text { Double-blind/ } \\
\text { parallel }\end{array}$ & $39 / 29$ & $62 \cdot 4$ & NR & 300 & Capsule & 24 & $\begin{array}{l}\text { TC: } 5 \cdot 6 / 5 \cdot 7 \\
\text { LDL: - } \\
\text { HDL: - } \\
\text { TAG: - }\end{array}$ & $\begin{array}{l}\text { TC: } 5 \cdot 6 / 5 \cdot 6 \\
\text { LDL: - } \\
\text { HDL: - } \\
\text { TAG: - }\end{array}$ & 4 \\
\hline Sano et al. ${ }^{(27)}$ & Japan & $\begin{array}{l}\text { Single-blind/ } \\
\text { parallel }\end{array}$ & $36 / 18$ & 52.9 & $24 \cdot 2$ & 400 & Tablet & 12 & $\begin{array}{l}\text { TC: } 5 \cdot 66 / 5 \cdot 84 \\
\text { LDL: } 3 \cdot 41 / 3 \cdot 56 \\
\text { HDL: } 1 \cdot 50 / 1 \cdot 66 \\
\text { TAG: } 1 \cdot 32 / 1 \cdot 38 \\
\text { TC: } 5 \cdot 66 / 5 \cdot 84 \\
\text { LDL: } 3 \cdot 41 / 3 \cdot 56 \\
\text { HDL: } 1.50 / 1 \cdot 66 \\
\text { TAG: } 1.32 / 1 \cdot 38\end{array}$ & $\begin{array}{l}\text { TC: } 5 \cdot 84 / 5 \cdot 81 \\
\text { LDL: } 3 \cdot 46 / 3 \cdot 46 \\
\text { HDL: } 1 \cdot 63 / 1 \cdot 77 \\
\text { TAG: } 1 \cdot 30 / 1 \cdot 25 \\
\text { TC: } 5 \cdot 74 / 5 \cdot 92 \\
\text { LDL: } 3 \cdot 41 / 3.56 \\
\text { HDL: } 1 \cdot 58 / 1 \cdot 73 \\
\text { TAG: } 1.55 / 1.46\end{array}$ & 2 \\
\hline Kar et al. ${ }^{(16)}$ & UK & $\begin{array}{l}\text { Double-blind/ } \\
\text { crossover }\end{array}$ & $64 / 32$ & 61.8 & $30 \cdot 2$ & 600 & Tablet & 4 & $\begin{array}{l}\text { TC: } 4 \cdot 4 / 4 \cdot 3 \\
\text { LDL: }- \\
\text { HDL: } 1 \cdot 2 / 1 \cdot 2 \\
\text { TAG: } 1 \cdot 7 / 1 \cdot 8\end{array}$ & $\begin{array}{l}\text { TC: } 4 \cdot 5 / 4 \cdot 3 \\
\text { LDL: }- \\
\text { HDL: } 1 \cdot 2 / 1 \cdot 2 \\
\text { TAG: } 1 \cdot 9 / 1 \cdot 7\end{array}$ & 3 \\
\hline $\begin{array}{l}\text { Sivaprakasapillai } \\
\text { et al. }{ }^{(29)}\end{array}$ & USA & $\begin{array}{l}\text { Single-blind/ } \\
\text { parallel }\end{array}$ & $27 / 9$ & 45 & 36 & 300 & Capsule & 4 & $\begin{array}{l}\text { TC: } 5 \cdot 14 / 5 \cdot 09 \\
\text { LDL: } 3 \cdot 23 / 3 \cdot 20 \\
\text { HDL: } 1 \cdot 31 / 1 \cdot 26 \\
\text { TAG: } 2 \cdot 02 / 2 \cdot 07 \\
\text { TC: } 5 \cdot 14 / 5 \cdot 09 \\
\text { LDL: } 3 \cdot 23 / 3 \cdot 20 \\
\text { HDL: } 1 \cdot 31 / 1 \cdot 26 \\
\text { TAG: } 2 \cdot 02 / 2 \cdot 07\end{array}$ & $\begin{array}{l}\text { TC: } 5 \cdot 58 / 5 \cdot 40 \\
\text { LDL: } 3 \cdot 80 / 3 \cdot 49 \\
\text { HDL: } 1 \cdot 31 / 1 \cdot 24 \\
\text { TAG: } 1 \cdot 99 / 2 \cdot 01 \\
\text { TC: } 5 \cdot 01 / 5 \cdot 06 \\
\text { LDL: } 3 \cdot 25 / 3 \cdot 31 \\
\text { HDL: } 1 \cdot 13 / 1 \cdot 16 \\
\text { TAG: } 2 \cdot 01 / 1.99\end{array}$ & 4 \\
\hline Yubero et al.(26) & Spain & $\begin{array}{l}\text { Double-blind/ } \\
\text { parallel }\end{array}$ & $60 / 30$ & 51 & $26 \cdot 9$ & 700 & Capsule & $>8$ & $\begin{array}{l}\text { TC: } 6 \cdot 53 / 6 \cdot 35 \\
\text { LDL: } 4 \cdot 35 / 4 \cdot 27 \\
\text { HDL: } 1 \cdot 19 / 1 \cdot 20 \\
\text { TAG: - }\end{array}$ & $\begin{array}{l}\text { TC: } 6 \cdot 39 / 5 \cdot 52 \\
\text { LDL: } 4 \cdot 31 / 3 \cdot 76 \\
\text { HDL: } 1 \cdot 14 / 1 \cdot 21 \\
\text { TAG: - }\end{array}$ & 3 \\
\hline
\end{tabular}


24 weeks. Ten trials reported mean age of participants, which varied between 44 and 62 years ${ }^{(13,15,16,24-30)}$. In addition, seven studies, out of eleven trials, were conducted among individuals with a mean $B M I \geq 27 \mathrm{~kg} / \mathrm{m}^{2(13,14,16,24,25,29,30)}$; others were carried out among subjects with a mean BMI $<27 \mathrm{~kg} / \mathrm{m}^{2}$. Six studies were done on hyperlipidaemic patients ${ }^{(14-16,24,28,29)}$ and others on normolipidaemic subjects. Furthermore, out of eleven trials, one study was conducted among subjects with breast cancer ${ }^{(30)}$, seven studies were done on individuals with high risk of CVD $^{(14-16,24,25,28,29)}$ and other three were done among healthy participants ${ }^{(13,26,27)}$. The method of intervention was using tablets or capsules in nine studies ${ }^{(13-16,26-30)}$ and functional foods in two researches ${ }^{(24,25)}$. Dosages of GSE used in these studies were different from 150 to $2000 \mathrm{mg} / \mathrm{d}$. Regarding the study quality, presented in Table 1 , one publication had a Jadad score of $2^{(27)}$ and others had a score of $\geq 3^{(13-16,24-26,28-30)}$.

\section{Findings from the meta-analysis}

All of the eleven trials included in the systematic review were also considered in the present meta-analysis, out of which levels of TC, LDL, HDL and TAG were evaluated in eleven, nine, ten and eight studies, respectively.

Combining thirteen effect sizes from eleven studies, we found that GSE supplementation did not significantly influence serum levels of TC $(-0.18 \mathrm{mmol} / 1 ; 95 \%$ CI $-0.38,0.03$; Fig. 2(a)). Stratification by the health condition of the participants did not change this finding (Fig. 2(b)). A significant between-study heterogeneity was observed (Cochran's $Q, \quad P<0.001$, $I^{2}=98.3 \%$ ). To investigate the potential sources of inter-study heterogeneity, we conducted subgroup analyses based on the country of origin, study design, health status of subjects, mean age and BMI of participants at study baseline, sex, and sample size, the type and dose of GSE supplementation as well as the duration of intervention (Table 2). In these analyses, we found that GSE supplementation resulted in decreased levels of TC among participants with a BMI of $<27 \mathrm{~kg} / \mathrm{m}^{2}$ compared with control group ( $-0.40 \mathrm{mmol} / 1 ; 95 \% \mathrm{CI}-0.72,-0 \cdot 07)$. In addition, GSE supplementation had favourable effects on TC levels in a subgroup of studies which used capsules as their intervention type $(-0.28 \mathrm{mmol} / 1 ; 95 \% \mathrm{CI}-0.53,-0.03)$ as well as those that had used the dosage of $<300 \mathrm{mg} / \mathrm{d} \quad(-0.30 \mathrm{mmol} / \mathrm{l}$; $95 \% \mathrm{CI}-0.55,-0.06)$, were done on a sample of more than fifty individuals $(-0.40 \mathrm{mmol} / \mathrm{l} ; 95 \% \mathrm{CI}-0.72,-0 \cdot 07)$, with a duration of $<10$ weeks $(-0.28 \mathrm{mmol} / \mathrm{l} ; 95 \%$ CI $-0 \cdot 54,-0 \cdot 03)$ (Table 2).

Pooling eleven effect sizes from nine studies revealed that GSE supplementation significantly reduced circulating LDL levels $(-0 \cdot 17 \mathrm{mmol} / 1 ; 95 \% \mathrm{CI}-0 \cdot 34,-0 \cdot 01$; Fig. 3(a)). Stratification by the health condition of the participants changed this finding (Fig. 3(b)). A significant between-study heterogeneity was found (Cochran's $Q, P<0.001, I^{2}=98.4 \%$ ). In the subgroup analyses, we found a significant LDL-lowering effect of GSE supplementation in studies performed on participants with a BMI of $<27 \mathrm{~kg} / \mathrm{m}^{2}(-0.33 \mathrm{mmol} / 1$; $95 \% \mathrm{CI}-0.56,-0 \cdot 10)$, those that used capsules as their intervention type $(-0.34 \mathrm{mmol} / \mathrm{l}$; $95 \%$ CI $-0.51,-0 \cdot 16)$, used the dosage of $<300 \mathrm{mg} / \mathrm{d}$ $(-0.30 \mathrm{mmol} / \mathrm{l} ; 95 \% \mathrm{CI}-0.54,-0.06)$, studies that were of 
(a)

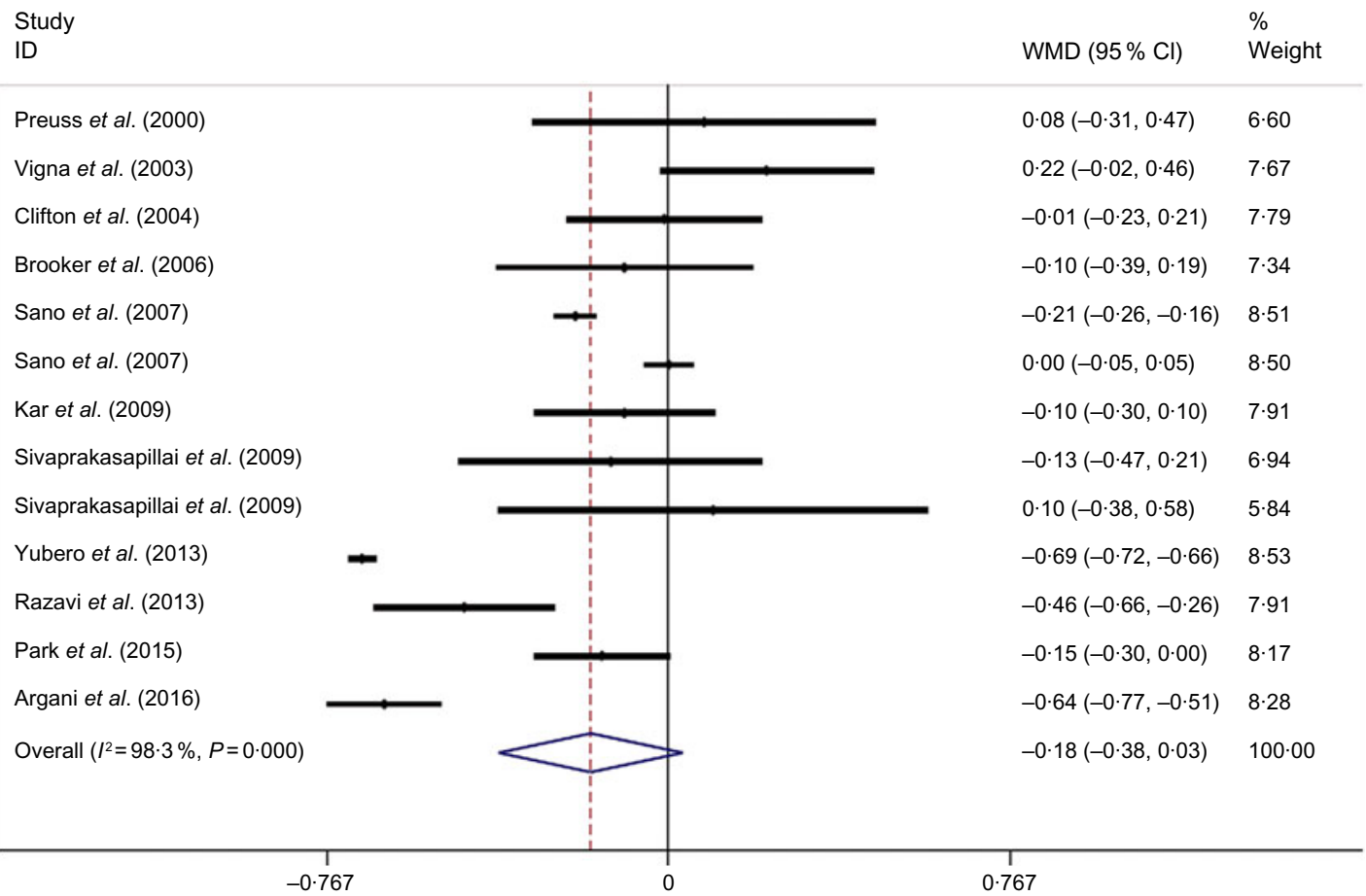

(b)

Study

ID

WMD $(95 \% \mathrm{Cl}) \quad$ Weight

\section{Healthy}

Vigna et al. (2003)

Sano et al. (2007)

Sano et al. (2007)

Yubero et al. (2013)

Subtotal $\left(I^{2}=99.5 \%, P=0.000\right)$

Chronic condition

Preuss et al. (2000)

Clifton et al. (2004)

Brooker et al. (2006)

Kar et al. (2009)

Sivaprakasapillai et al. (2009)

Sivaprakasapillai et al. (2009)

Razavi et al. (2013)

Park et al. (2015)

Argani et al. (2016)

Subtotal $\left(I^{2}=85.0 \%, P=0.000\right)$

Overall $\left(I^{2}=98.3 \%, P=0.000\right)$
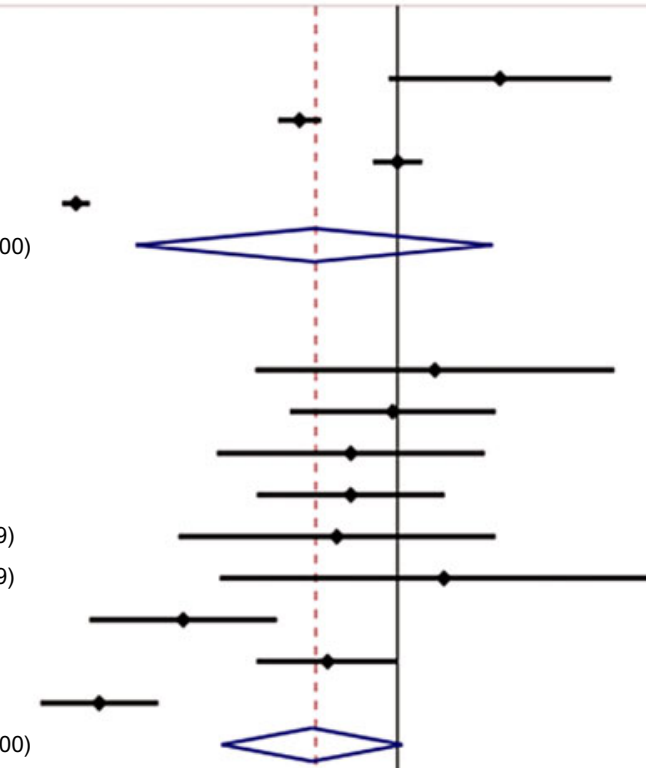

$-0.01(-0.23,0.21) \quad 7.79$

$-0.10(-0.39,0 \cdot 19) \quad 7.34$

$-0 \cdot 10(-0.30,0 \cdot 10) \quad 7.91$

$-0.13(-0.47,0.21) \quad 6.94$

$0.10(-0.38,0.58) \quad 5.84$

$-0.46(-0.66,-0.26) \quad 7.91$

$-0.15(-0.30,0.00) \quad 8.17$

$-0.64(-0.77,-0.51) \quad 8.28$

$-0.18(-0.38,0.01) \quad 66.78$

$-0.18(-0.38,0.03) \quad 100 \cdot 00$

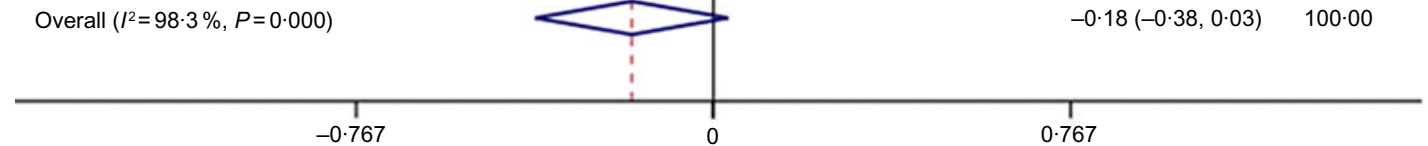

Fig. 2. Forest plot for the effect of grape seed extract supplementation on serum levels of total cholesterol using a random effects model in all participants (a) and stratified by the health condition of participants (b). Weights are from random effects analysis. 
Table 2. Subgroup analysis based on random effects models of grape seed extract (GSE) supplementation on plasma lipids (Weighted mean difference (WMD), $95 \%$ confidence intervals and $l^{2}$ )

\begin{tabular}{|c|c|c|c|c|c|c|c|c|c|c|c|c|}
\hline \multirow[b]{2}{*}{ Subgroups } & \multicolumn{3}{|c|}{ Total cholesterol } & \multicolumn{3}{|c|}{ LDL } & \multicolumn{3}{|c|}{ HDL } & \multicolumn{3}{|c|}{ TAG } \\
\hline & WMD & $95 \% \mathrm{Cl}$ & $I^{2}(\%)$ & WMD & $95 \% \mathrm{Cl}$ & $I^{2}(\%)$ & WMD & $95 \% \mathrm{Cl}$ & $I^{2}(\%)$ & WMD & $95 \% \mathrm{Cl}$ & $I^{2}(\%)$ \\
\hline Overall & -0.18 & $-0.38,0.03$ & $98 \cdot 3$ & -0.17 & $-0.34,-0.01$ & $98 \cdot 4$ & 0.01 & $-0.03,0.04$ & 88.6 & -0.11 & $-0.18,-0.05$ & 73.4 \\
\hline \multicolumn{13}{|l|}{ Country } \\
\hline USA & -0.11 & $-0.23,0.02$ & 0.0 & -0.01 & $-0.11,0.09$ & 0.0 & -0.01 & $-0.09,0.06$ & 61.6 & -0.08 & $-0.17,0.01$ & 0.0 \\
\hline Non-USA & -0.23 & $-0.47,0.02$ & $98 \cdot 8$ & -0.23 & $-0.43,-0.04$ & $99 \cdot 0$ & 0.01 & $-0.03,0.05$ & 90.2 & -0.13 & $-0.21,-0.04$ & 81.6 \\
\hline \multicolumn{13}{|l|}{ Study design } \\
\hline Parallel & -0.21 & $-0.47,0.04$ & 98.8 & -0.20 & $-0.40,-0.00$ & $98 \cdot 9$ & 0.01 & $-0.03,0.06$ & $91 \cdot 0$ & -0.15 & $-0.22,-0.08$ & $69 \cdot 0$ \\
\hline Crossover & -0.09 & $-0.37,0.18$ & $84 \cdot 7$ & -0.10 & $-0.41,0.21$ & $88 \cdot 3$ & -0.01 & $-0.05,0.03$ & 33.8 & -0.04 & $-0.23,0.15$ & $79 \cdot 6$ \\
\hline \multicolumn{13}{|c|}{ Study duration (weeks) } \\
\hline$<10$ & -0.28 & $-0.54,-0.03$ & $92 \cdot 5$ & -0.35 & $-0.56,-0.14$ & $84 \cdot 0$ & 0.04 & $0.00,0.09$ & $46 \cdot 0$ & -0.21 & $-0.37,-0.05$ & $58 \cdot 2$ \\
\hline$\geq 10$ & -0.11 & $-0.24,0.02$ & $88 \cdot 8$ & -0.08 & $-0.19,0.02$ & $91 \cdot 0$ & -0.02 & $-0.04,-0.00$ & $40 \cdot 2$ & -0.08 & $-0.15,-0.02$ & $67 \cdot 0$ \\
\hline \multicolumn{13}{|l|}{ Total sample size } \\
\hline$<50$ & -0.05 & $-0.17,0.07$ & 83.9 & -0.04 & $-0.14,0.06$ & $84 \cdot 2$ & -0.02 & $-0.04,0.01$ & $35 \cdot 1$ & -0.07 & $-0.15,0.01$ & 66.9 \\
\hline$\geq 50$ & -0.39 & $-0.64,-0.15$ & $94 \cdot 3$ & -0.40 & $-0.58,-0.23$ & $90 \cdot 0$ & 0.03 & $-0.03,0.09$ & $86 \cdot 1$ & $-0 \cdot 19$ & $-0.35,-0.04$ & 81.5 \\
\hline \multicolumn{13}{|l|}{ Sex } \\
\hline Male & 0.22 & $-0.02,0.46$ & - & -0.11 & $-0.11,0.33$ & - & 0.00 & $-0.07,0.07$ & - & 0.28 & $0.07,0.49$ & - \\
\hline Both & -0.22 & $-0.44,0.00$ & 98.5 & - & & & 0.01 & $-0.03,0.04$ & 89.5 & $-0 \cdot 14$ & $-0.20,-0.09$ & $59 \cdot 8$ \\
\hline \multicolumn{13}{|l|}{ Age (years) } \\
\hline$<50$ & -0.24 & $-0.49,0.01$ & $86 \cdot 4$ & -0.23 & $-0.51,0.06$ & $92 \cdot 5$ & -0.00 & $-0.06,0.06$ & $68 \cdot 5$ & $-0 \cdot 14$ & $-0.26,-0.03$ & 74.9 \\
\hline$\geq 50$ & $-0 \cdot 13$ & $-0.42,0.16$ & $99 \cdot 1$ & -0.12 & $-0.35,0.12$ & $99 \cdot 3$ & 0.01 & $-0.03,0.05$ & $90 \cdot 3$ & -0.08 & $-0.18,0.02$ & 76.9 \\
\hline \multicolumn{13}{|l|}{$\mathrm{BM} \overline{\mathrm{M}}\left(\mathrm{kg} / \mathrm{m}^{2}\right)$} \\
\hline$<27$ & -0.40 & $-0.72,-0.07$ & $99 \cdot 4$ & -0.33 & $-0.56,-0.10$ & $99 \cdot 3$ & 0.01 & $-0.04,0.07$ & $94 \cdot 1$ & $-0 \cdot 16$ & $-0.24,-0.09$ & 80.9 \\
\hline$\geq 27$ & -0.04 & $-0.14,0.05$ & $13 \cdot 0$ & 0.00 & $-0.08,0.08$ & 0.0 & -0.00 & $-0.04,0.04$ & $45 \cdot 7$ & -0.04 & $-0 \cdot 18,0 \cdot 10$ & $64 \cdot 3$ \\
\hline \multicolumn{13}{|l|}{ GSE dosage $(\mathrm{mg} / \mathrm{d})$} \\
\hline$<300$ & -0.30 & $-0.55,0.06$ & 91.4 & -0.30 & $-0.54,-0.06$ & 93.8 & -0.02 & $-0.06,0.03$ & $51 \cdot 8$ & $-0 \cdot 16$ & $-0.26,-0.05$ & $81 \cdot 3$ \\
\hline$\geq 300$ & $-0 \cdot 10$ & $-0.43,0.23$ & $98 \cdot 8$ & -0.06 & $-0.34,0.21$ & $99 \cdot 0$ & 0.01 & $-0.03,0.05$ & 89.6 & -0.07 & $-0.19,0.05$ & 71.6 \\
\hline \multicolumn{13}{|c|}{ GSE supplementation type } \\
\hline Tablet & -0.08 & $-0.24,0.07$ & 91.4 & -0.06 & $-0.19,0.08$ & $93 \cdot 6$ & -0.01 & $-0.03,0.00$ & 0.0 & -0.14 & $-0.19,-0.08$ & $34 \cdot 9$ \\
\hline Capsule & -0.28 & $-0.53,-0.03$ & $93 \cdot 6$ & -0.34 & $-0.51,-0.16$ & $87 \cdot 8$ & 0.03 & $-0.03,0.09$ & $83 \cdot 3$ & -0.07 & $-0.25,0.11$ & $86 \cdot 5$ \\
\hline Functional food & $-0 \cdot 10$ & $-0.23,0.03$ & 4.4 & -0.00 & $-0.10,0.09$ & 0.0 & -0.02 & $-0.11,0.07$ & $80 \cdot 3$ & -0.08 & $-0.17,0.01$ & 0.0 \\
\hline \multicolumn{13}{|l|}{ Baseline status } \\
\hline Healthy & $-0 \cdot 18$ & $-0.56,0.20$ & $99 \cdot 5$ & -0.14 & $-0.40,0.13$ & 99.5 & 0.01 & $-0.04,0.06$ & $93 \cdot 9$ & -0.05 & $-0.17,0.07$ & $86 \cdot 2$ \\
\hline Chronic condition & $-0 \cdot 18$ & $-0.38,0.01$ & $85 \cdot 0$ & -0.20 & $-0.44,0.05$ & $98 \cdot 3$ & 0.00 & $-0.04,0.04$ & $68 \cdot 3$ & $-0 \cdot 15$ & $-0.24,-0.05$ & $67 \cdot 8$ \\
\hline No & $-0 \cdot 16$ & $-0.47,0.15$ & $99 \cdot 3$ & -0.11 & $-0.35,0.13$ & $99 \cdot 3$ & -0.00 & $-0.06,0.05$ & 88.6 & -0.07 & $-0.16,0.02$ & $79 \cdot 5$ \\
\hline
\end{tabular}

parallel design ( $-0.20 \mathrm{mmol} / 1 ; 95 \% \mathrm{CI}-0 \cdot 40,-0 \cdot 00)$, publications that were done on a sample size of more than fifty individuals $(-0.40 \mathrm{mmol} / \mathrm{l} ; 95 \% \mathrm{CI}-0 \cdot 58,-0 \cdot 23)$ and duration of $<10$ weeks $(-0.35 \mathrm{mmol} / \mathrm{l} ; 95 \% \mathrm{CI}-0.56,-0.14)$ and those came from non-USA countries $(-0.23 \mathrm{mmol} / \mathrm{l} ; 95 \% \mathrm{CI}-0.43,-0.04)$.

Combining twelve effect sizes from ten studies, we found that GSE supplementation did not influence serum HDL levels (0.01 mmol/1; $95 \%$ CI $-0.03,0 \cdot 04$; Fig. 4(a)). Stratification by the health condition of the participants did not change this finding (Fig. 4(b)). There was a significant between-study heterogeneity (Cochran's $Q, P<0 \cdot 001, I^{2}=88.6 \%$ ). Potential sources of heterogeneity were assessed using subgroup analysis. As illustrated in Table 2, GSE supplementation resulted in an increment in serum HDL concentrations in studies with an intervention duration of $<10$ weeks ( $0.04 \mathrm{mmol} / 1 ; 95 \%$ CI 0.001, 0.09).

Pooling eleven effect sizes from eight studies, we found that GSE supplementation significantly decreased serum levels of TAG $(-0.11 \mathrm{mmol} / \mathrm{l} ; 95 \%$ CI $-0 \cdot 18,-0 \cdot 05$; Fig. 5(a)). Stratification by the health condition of the participants changed this finding (Fig. 5(b)). A significant between-study heterogeneity was observed (Cochran's $Q, P<0 \cdot 001, I^{2}=73.4 \%$ ). In subgroup analyses, we observed that GSE supplementation significantly reduced TAG levels in studies that were carried out among non-healthy participants $(-0.15 \mathrm{mmol} / \mathrm{l} ; 95 \% \mathrm{CI}-0.24$, $-0.05)$, those that were performed on participants with a BMI of $<27 \mathrm{~kg} / \mathrm{m}^{2}(-0.16 \mathrm{mmol} / \mathrm{l} ; 95 \% \mathrm{CI}-0.24,-0.09)$ as well as those that were done on people aged $<50$ years $(-0 \cdot 14 \mathrm{mmol} / \mathrm{l}$; $95 \%$ CI-0.26, -0.03). In addition, GSE supplementation had favourable effects on TAG levels in a subgroup of studies which used capsules as their intervention type $(-0.14 \mathrm{mmol} / \mathrm{l}$; $95 \% \mathrm{CI}-0 \cdot 19,-0 \cdot 08)$ as well as those that had used the dosage of $<300 \mathrm{mg} / \mathrm{d}(-0.16 \mathrm{mmol} / \mathrm{l} ; 95 \% \mathrm{CI}-0 \cdot 26,-0 \cdot 05)$, were done on a sample of more than fifty individuals $(-0.19 \mathrm{mmol} / \mathrm{l}$; $95 \%$ CI $-0 \cdot 35,-0 \cdot 04)$, publications that were of parallel design $(-0.15 \mathrm{mmol} / 1 ; 95 \% \mathrm{CI}-0.22,-0.08)$ and those came from non-USA countries $(-0 \cdot 13 \mathrm{mmol} / 1 ; 95 \% \mathrm{CI}-0 \cdot 21,-0 \cdot 04)$.

Findings from dose-response analysis about GSE supplementation on lipid profiles revealed that the overall pooled estimates on lipid profiles were independent of GSE dosage. We failed to detect a significant effect of specific dosage of GSE on lipid profiles, as examined by non-linear dose-response meta-analysis (Fig. 6). 
(a)

\begin{tabular}{|c|c|c|c|}
\hline $\begin{array}{l}\text { Study } \\
\text { ID }\end{array}$ & & WMD $(95 \% \mathrm{Cl})$ & $\begin{array}{l}\% \\
\text { Weight }\end{array}$ \\
\hline Preuss et al. (2000) & & $0.06(-0.24,0.36)$ & $7 \cdot 90$ \\
\hline Vigna et al. (2003) & & $0 \cdot 11(-0.11,0.33)$ & 8.97 \\
\hline Clifton et al. (2004) & & $-0.02(-0.22,0.18)$ & $9 \cdot 24$ \\
\hline Sano et al. (2007) & & $-0.15(-0 \cdot 18,-0 \cdot 12)$ & $10 \cdot 69$ \\
\hline Sano et al. (2007) & & $0.00(-0.04,0.04)$ & $10 \cdot 68$ \\
\hline Sivaprakasapillai et al. (2009) & & $-0.28(-0.65,0.09)$ & $7 \cdot 06$ \\
\hline Sivaprakasapillai et al. (2009) & & $0.09(-0.52,0.70)$ & $4 \cdot 35$ \\
\hline Yubero et al. (2013) & - & $-0.47(-0.49,-0.45)$ & $10 \cdot 73$ \\
\hline Razavi et al. (2013) & & $-0.37(-0.50,-0.24)$ & $10 \cdot 10$ \\
\hline Park et al. (2015) & & $0.00(-0 \cdot 11,0 \cdot 11)$ & $10 \cdot 27$ \\
\hline Argani et al. (2016) & & $-0.68(-0.82,-0.54)$ & $10 \cdot 01$ \\
\hline Overall $\left(I^{2}=98.4 \%, P=0.000\right)$ & & $-0.17(-0.34,-0.01)$ & $100 \cdot 00$ \\
\hline
\end{tabular}

(b)

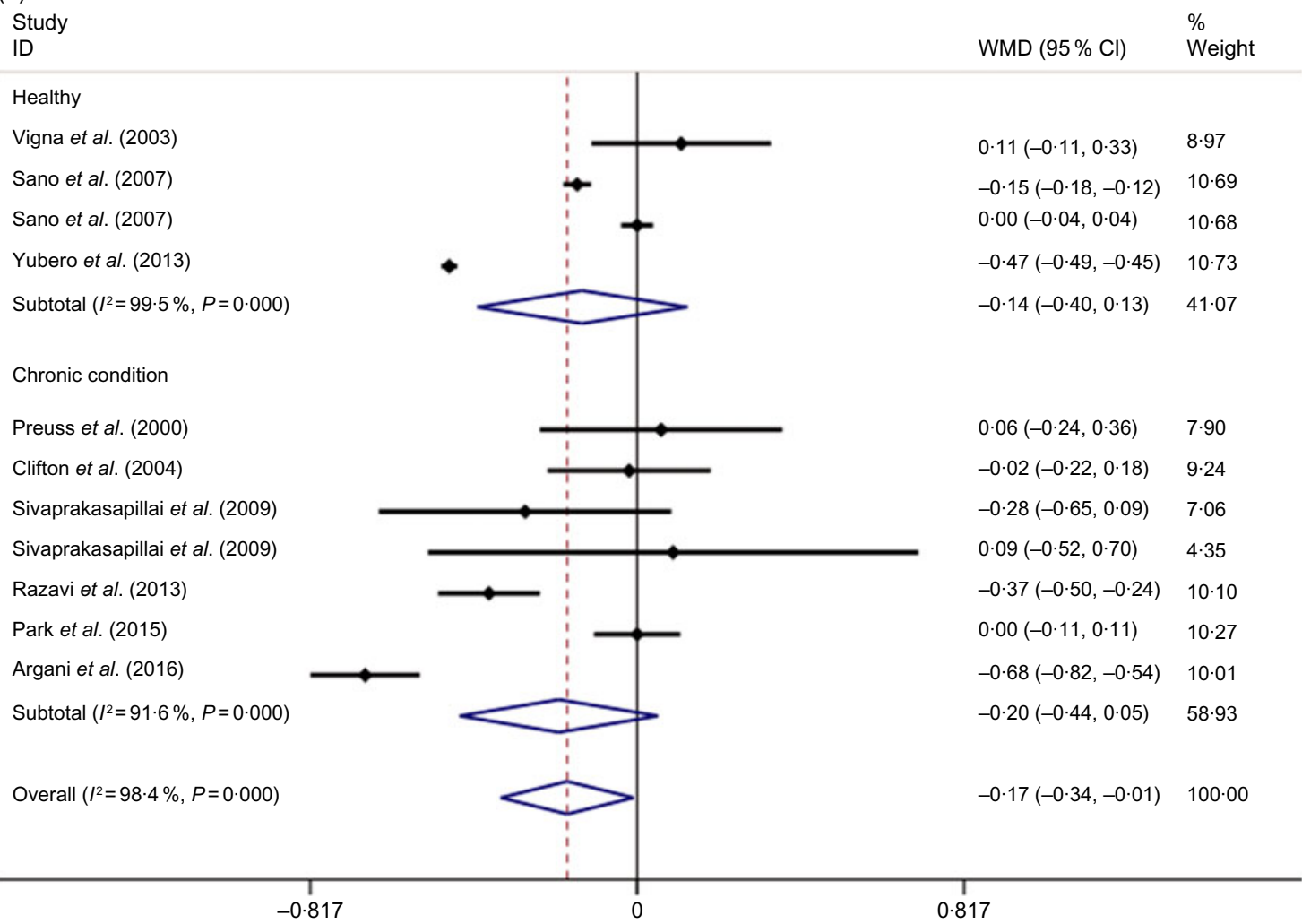

Fig. 3. Forest plot for the effect of grape seed extract supplementation on serum levels of LDL-cholesterol using a random effects model in all participants (a) and stratified by the health condition of participants (b). Weights are from random effects analysis.

For all lipid profiles, no evidence of publication bias was seen through visual inspection of funnel plots (online Supplementary Fig. 1). Moreover, these findings were also confirmed by the Egger's regression test (for TC: $P=0 \cdot 15$; LDL: $P=0 \cdot 28$;
HDL: $P=0 \cdot 07$; TAG: $P=0 \cdot 69$ ). In addition, sensitivity analysis demonstrated that excluding individual studies did not alter the estimated pooled effect sizes in lipid profiles (online Supplementary Fig. 2). 
(a)

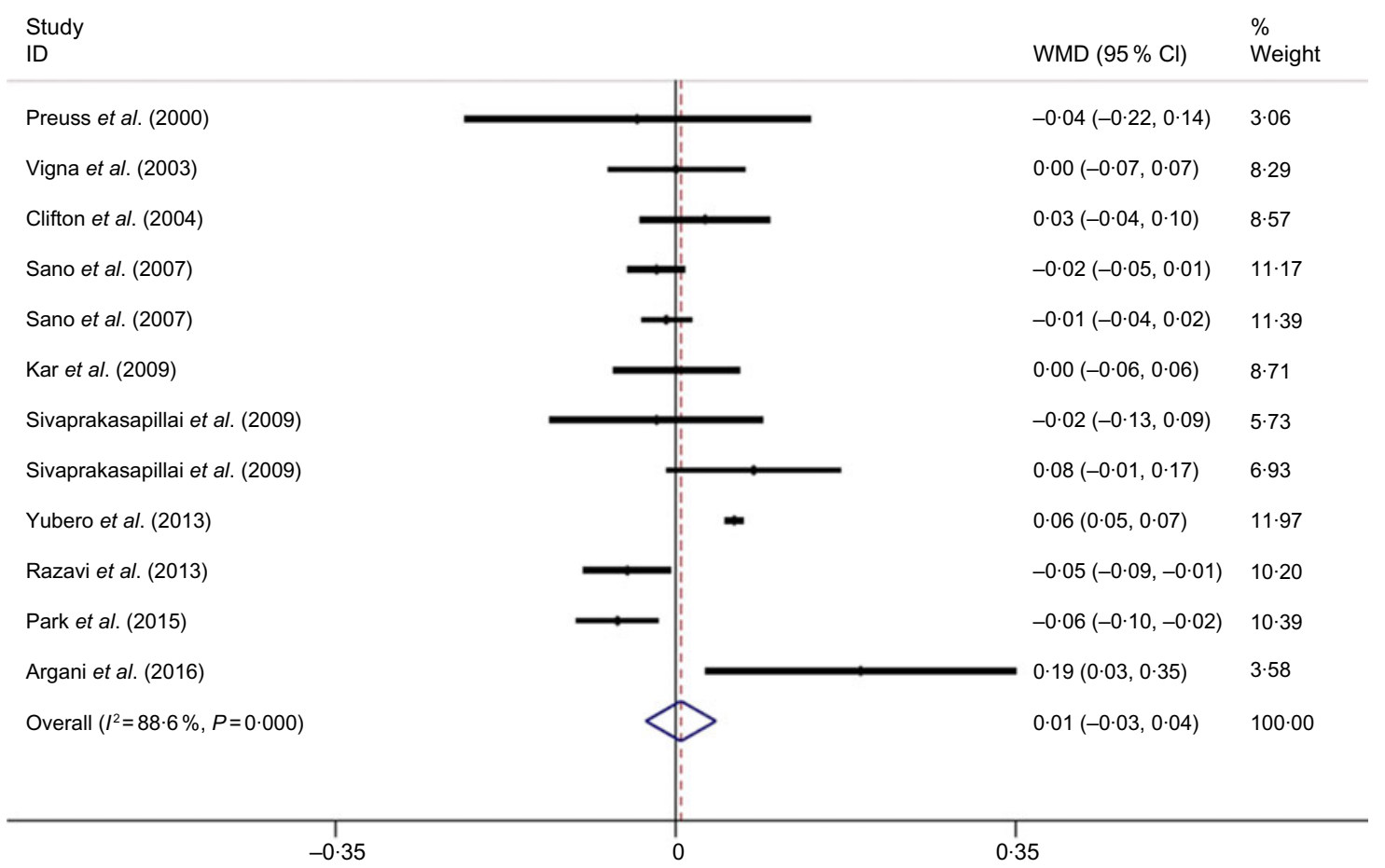

(b)

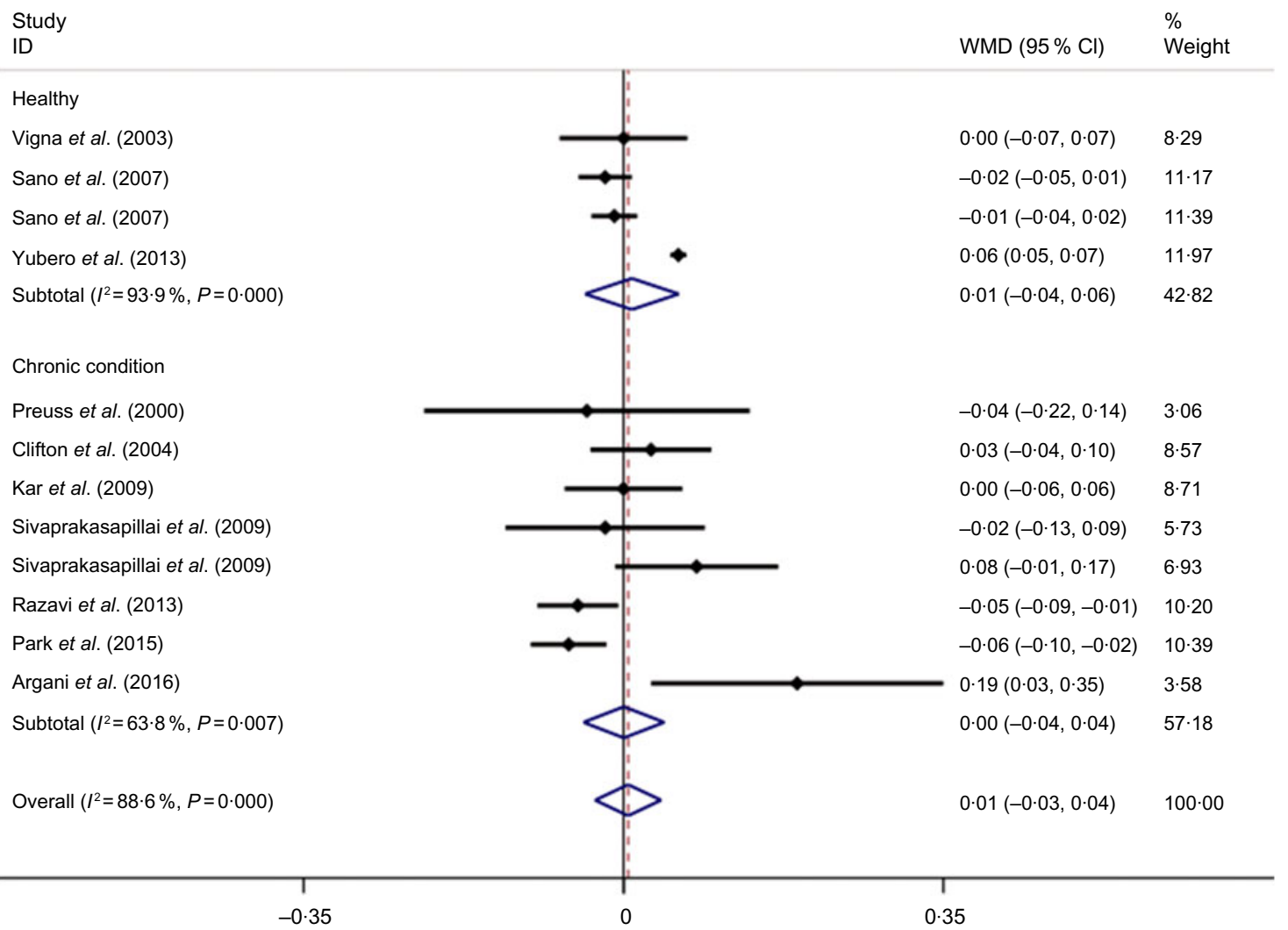

Fig. 4. Forest plot for the effect of grape seed extract supplementation on serum levels of HDL-cholesterol using a random effects model in all participants (a) and stratified by the health condition of participants (b). Weights are from random effects analysis. 
(a)

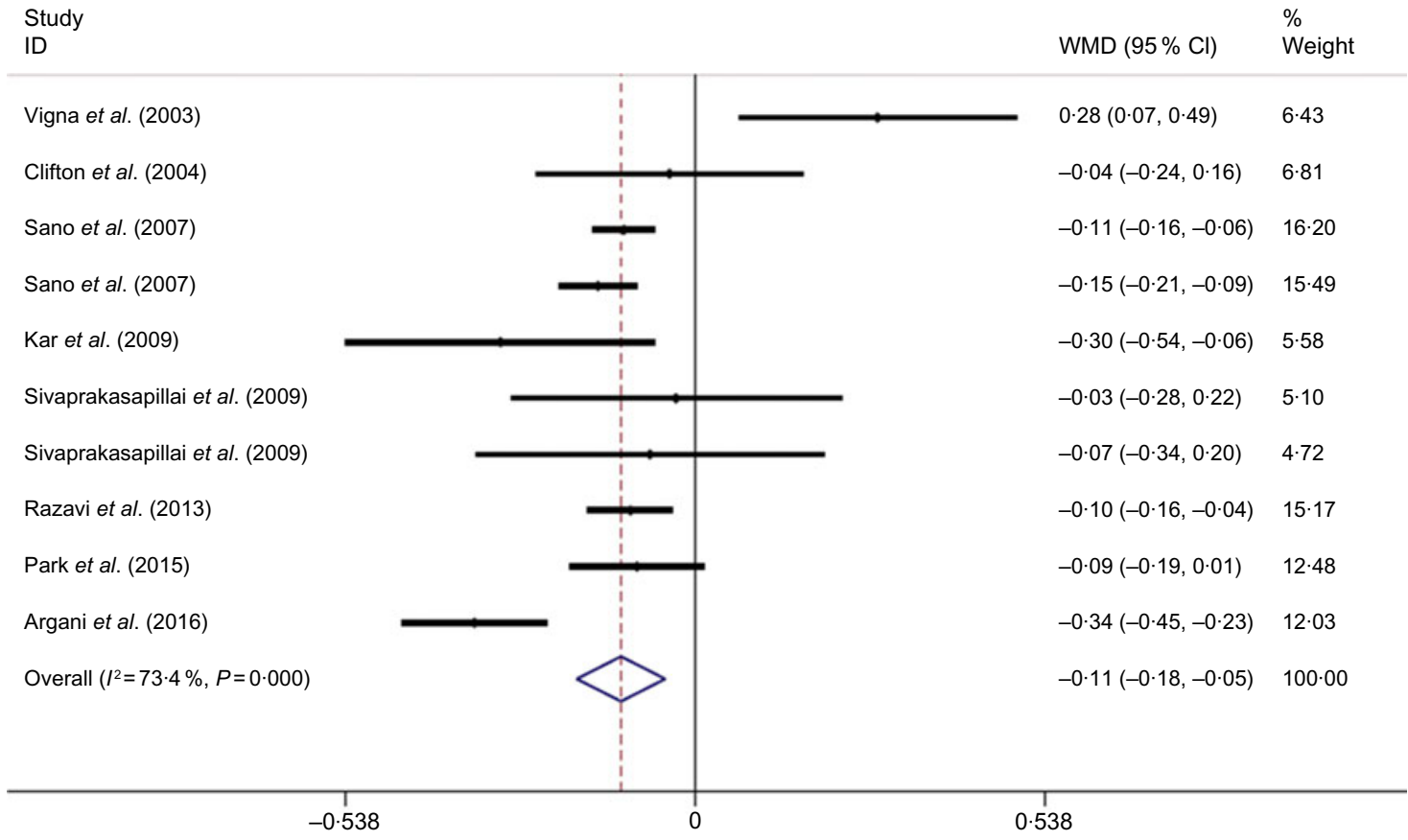

(b)

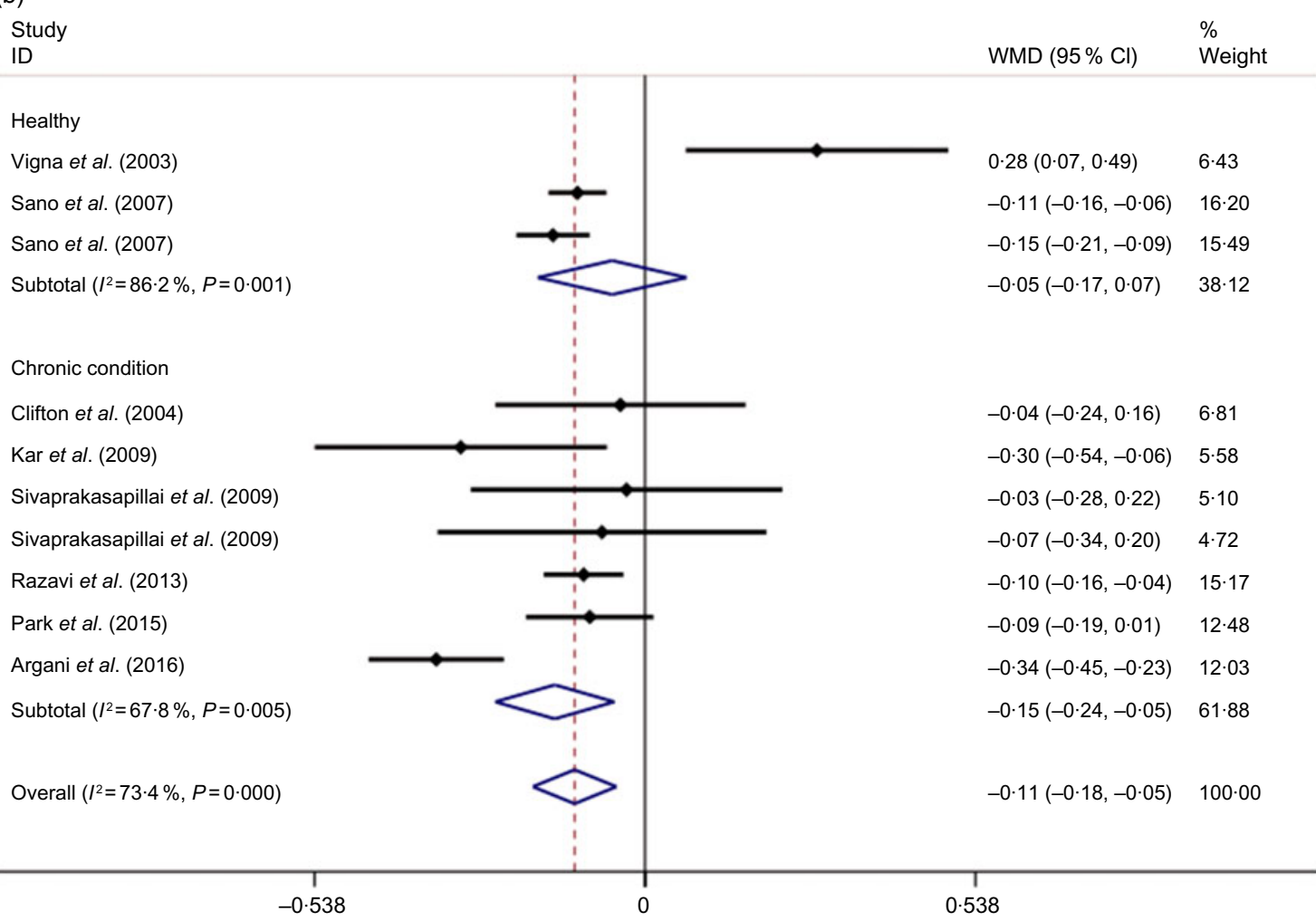

Fig. 5. Forest plot for the effect of grape seed extract supplementation on serum levels of TAG using a random effects model in all participants (a) and stratified by the health condition of participants (b). Weights are from random effects analysis.

\section{Discussion}

In the present meta-analysis of eleven trials, we observed that GSE supplementation resulted in a statistically significant reduction in serum levels of LDL-cholesterol and TAG, but it did not affect TC and HDL-cholesterol concentrations.
Although the efficacy of several medications, including statins, in lowering serum levels of LDL and reducing CHD events has been already established, finding a novel adjunct therapy with lower complications is still challenging. In the present meta-analysis, we found that GSE supplementation significantly 
(a)

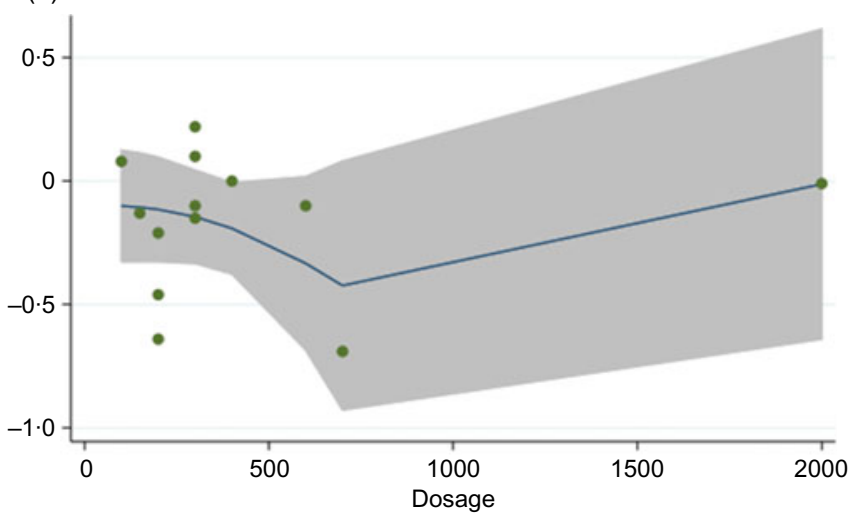

(c)

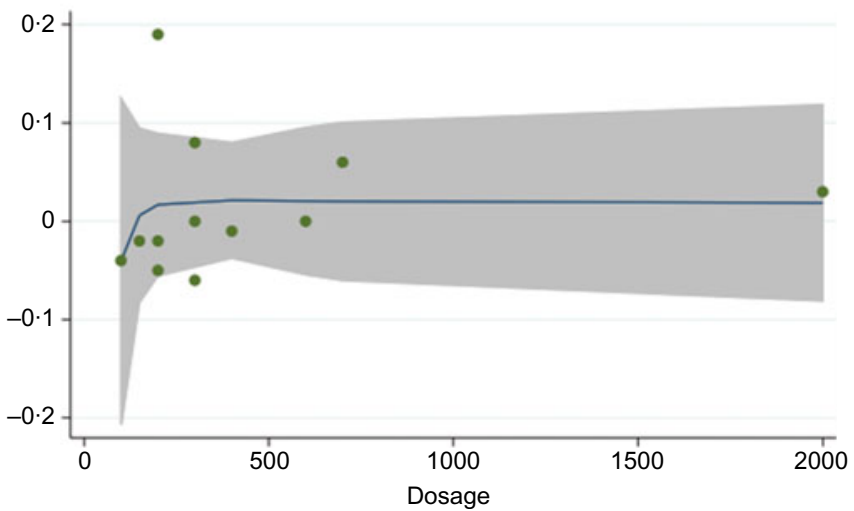

(b)

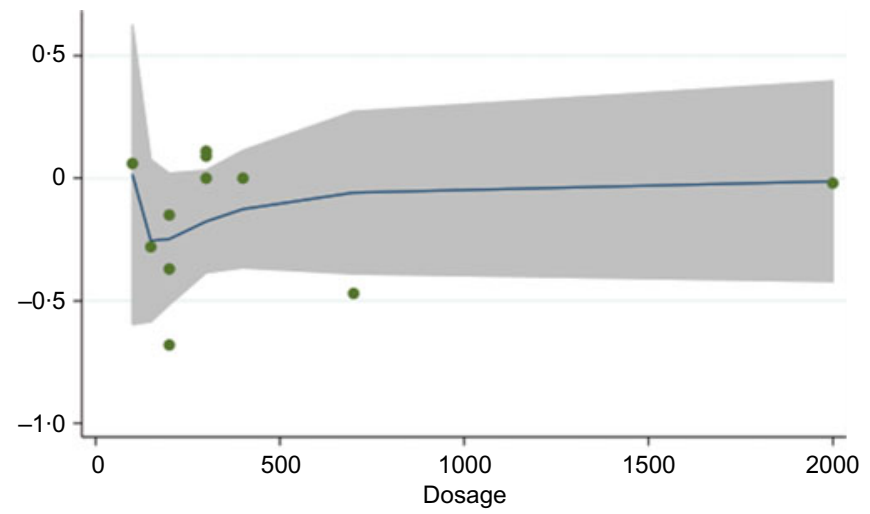

(d)

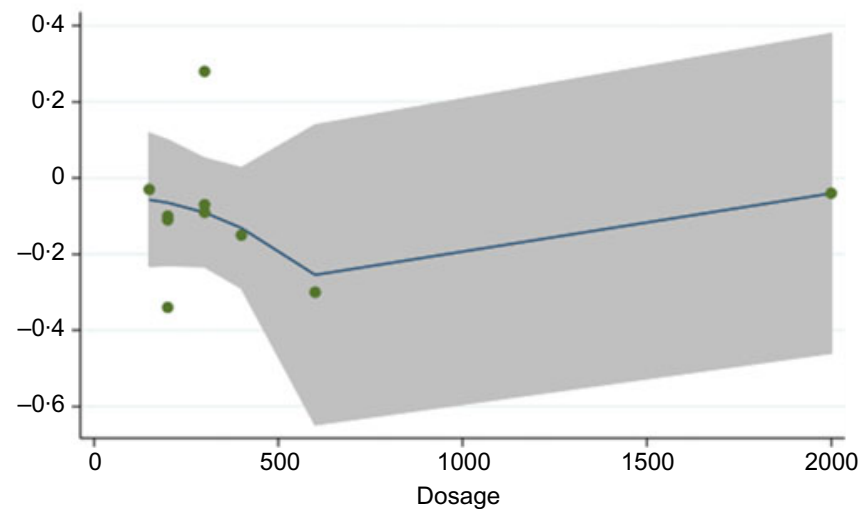

Fig. 6. Non-linear dose-response relationships between grape seed extract dosage (mg/d) and serum levels of lipids (mmol/l) in (a) total cholesterol, (b) LDLcholesterol, (c) HDL-cholesterol and (d) TAG. , predicted effect size; $\bullet$, weighted mean difference.

decreased serum levels of LDL. In addition to traditional lipid profiles, some studies have investigated the effect of GSE supplementation on postprandial lipid profiles. In line with our findings, a crossover trial on postprandial lipids demonstrated that GSE supplementation resulted in enhanced postprandial plasma antioxidant capacity and thereby decreased levels of oxidised LDL ${ }^{(31)}$. Similarly, Natella et al. reported that GSE supplementation remarkably reduced lipid peroxidation ${ }^{(32)}$. However, in a long-term semi-experimental study on seventeen people, GSE supplementation did not lead to any significant change in serum LDL concentrations in healthy individuals; however, it resulted in a significant reduction in LDL in hypercholesterolaemic participants ${ }^{(33)}$. This inconsistency in findings about the effect of GSE supplementation on LDL levels might be attributed to differences in the composition of polyphenolic extract, along with trials' designs and participants' conditions.

This meta-analysis revealed that GSE supplementation reduced serum levels of TAG. This finding was against previous studies about the effect of GSE on postprandial levels of TAG in both healthy and hypercholesterolaemic participants ${ }^{(33)}$. Experimental studies have shown that polyphenolic compounds regulate pathways involved in lipoprotein metabolism which can in turn result in reduced serum TAG concentrations ${ }^{(34)}$. These effects might be explained through alteration in microsomal transport protein activity and apoB secretion ${ }^{(35)}$. For instance, Pal et al. detected the efficacy of grape polyphenols on lipoprotein production and clearance in cultured liver cells ${ }^{(36)}$. In addition, the present study showed a significant reduction in TAG concentrations following GSE supplementation only among patients with chronic diseases. However, due to limited number of included studies and insufficient data about each disease, further studies are required to reach a firm conclusion in this area. Although the lipid-lowering effects of the polyphenols are well established in vitro, their in vivo effects are less documented. These compounds in GSE are extensively conjugated, and only a minor fraction remains unconjugated, thereby making it difficult to link biological effects to structure. This is particularly evident in the case of grape polyphenols which are mostly monomeric and their bioavailability is often underestimated due to poor detection and absorption $^{(37,38)}$. However, consumption of other dietary sources of flavonoids, including dark chocolate and cocoa powder, has been shown to significantly influence serum TAG and LDLcholesterol concentrations ${ }^{(39,40)}$. For instance, in a meta-analysis of ten randomised trials involving 320 individuals, dark chocolate consumption led to a significant reduction in serum LDLcholesterol concentrations in subjects with CVD risk factors ${ }^{(41)}$. Moreover, the study of Jia et al. revealed that short-term consumption of a cocoa product lowered serum levels of LDL-cholesterol and $\mathrm{TC}^{(42)}$. In addition, a growing body of evidence from both in vitro studies and animal studies also demonstrated the antidyslipidaemic and anti-inflammatory effects of cocoa and cocoa flavonoids ${ }^{(43-45)}$. These favourable effects on the lipid 
profile might be explained by the reduction in the hepatic activity of 3-hydroxy-3-methlyglutaryl-coenzyme reductase, the limiting enzyme of cholesterogenesis ${ }^{(46)}$.

The results of the present meta-analysis suggest that there is no significant effect of GSE supplementation on circulating TC and HDL-cholesterol levels. These findings were opposite to those reported from experimental studies. For instance, Vinson et al. reported that GSE induced a pronounced reduction in serum cholesterol levels in an atherosclerotic hamster model $^{(47)}$. Additionally, $28 \mathrm{~d}$ of GSE administration in rats reduced serum levels of lipids and prevented occurrences of fatty liver ${ }^{(48)}$. In vivo experiments have also revealed that GSE supplementation might increase cholesterol exertion through reducing intestinal absorption ${ }^{(49,50)}$. The difference between human studies and those done on animals might be explained by the dosage of supplementation, duration of intervention, physiological difference and different study designs.

The lipid-lowering impacts of GSE were more evident in trials with $<10$ weeks of intervention. For instance, although for whole studies combined, we did not observe any significant effect of GSE supplementation on serum TC and HDL-cholesterol levels, the significant effect on these lipids was seen for studies with $<10$ weeks of intervention. Besides study duration, GSE dosages might also play a role in this regard. It seems that the dosages of $<300 \mathrm{mg}$ of GSE per d are more officious than higher doses to influence blood lipids. Another point is the significant effect of GSE on lipid profiles among subjects with a BMI of $<27 \mathrm{~kg} / \mathrm{m}^{2}$. Some of these discrepancies might be explained by differences in composition of GSE products. Moreover, different characteristics of populations being studied might also provide some reasons. Previous investigations have demonstrated that differences in gut microbiota can result in large inter-individual variability in plasma concentrations of all phenolic acids ${ }^{(51,52)}$.

Although the precise mechanisms of GSE on blood lipids remain unclear, the beneficial effects might be attributed to modulation of antioxidant enzymes' expression, protection against oxidative damage in cells, antiatherosclerotic and antiinflammatory effects ${ }^{(53)}$. These effects were specifically attributed to proanthocyanidins in GSE. These compounds may also reduce plasma lipid profiles by inhibiting specific cholesterol transporters such as the Niemann-Pick C1-like one cholesterol transporter ${ }^{(54)}$. In addition, inhibition of pancreatic lipase, cholesterol esterase, cholesterol micellisation and bile acid binding is among other lipid-lowering mechanisms of $\mathrm{GSE}^{(12)}$. Moreover, GSE supplementation has been indicated to suppress intestinal lipid absorption, chylomicron and VLDL secretion, and subsequently reduced lipid levels ${ }^{(49)}$. The polyphenolic compounds of GSE are extremely varied which can explain the betweenstudy heterogeneity. We found that GSE had no significant effect on lipids when administered in high dosages and for long time. It should be noted that GSE is a rich source of fatty acids, including SFA and PUFA. Therefore, it is possible that long-term intake of high dosage of GSE might neutralise its beneficial effects on lipids.

This is a comprehensive up-to-date meta-analysis that examined the effect of GSE supplementation on circulating lipid concentrations. However, several limitations should be considered. First, the sample size of included studies was not sufficiently large to detect significant effects. The effect of different forms of GSE supplements was not adequately examined, and further investigations are warranted to address questions specific to efficacy, bioavailability and complete metabolite profiles.

In conclusion, GSE supplementation seems to favourably affect serum levels of LDL as well as TAG levels, but it did not influence TC and HDL-cholesterol concentrations. However, given the limitations and small sample sizes of included studies, further investigations are needed to shed light on this issue. The take-home message of this study would be the recommendation to administer GSE as a secondary factor, along with medications, to control hyperlipidaemia.

\section{Acknowledgements}

The authors would like to thank the authorities in the Tehran University of Medical Sciences for financial support of the study.

This study was supported by the School of Nutritional Sciences and Dietetics, Tehran University of Medical Sciences, Tehran, Iran.

J. A.-S., A. M., B. L. and A. E. contributed in conception, design, statistical analysis, data interpretation and manuscript drafting. All authors approved the final manuscript for submission.

The authors declare that there are no conflicts of interest.

\section{Supplementary material}

For supplementary material referred to in this article, please visit https://doi.org/10.1017/S0007114520000902

\section{References}

1. Hertog MG, Feskens EJ, Hollman PC, et al. (1993) Dietary antioxidant flavonoids and risk of coronary heart disease: the Zutphen Elderly Study. Lancet 342, 1007-1011.

2. Corder R, Mullen W, Khan NQ, et al. (2006) Oenology: red wine procyanidins and vascular health. Nature 444, 566.

3. Sánchez-Moreno C, Cao G, Ou B, et al. (2003) Anthocyanin and proanthocyanidin content in selected white and red wines. Oxygen radical absorbance capacity comparison with nontraditional wines obtained from highbush blueberry. J Agric Food Chem 51, 4889-4896.

4. Shi J, Yu J, Pohorly JE, et al. (2003) Polyphenolics in grape seeds - biochemistry and functionality. JMed Food 6, 291-299.

5. Viana M, Barbas C, Bonet B, et al. (1996) In vitro effects of a flavonoid-rich extract on LDL oxidation. Atherosclerosis 123, 83-91.

6. Frankel EN, Kanner J, German JB, et al. (1993) Inhibition of oxidation of human low-density lipoprotein by phenolic substances in red wine. Lancet 341, 454-457.

7. Miyagi Y, Miwa K \& Inoue H (1997) Inhibition of human low-density lipoprotein oxidation by flavonoids in red wine and grape juice. Am J Cardiol 80, 1627-1631.

8. Aviram M \& Fuhrman B (1998) Polyphenolic flavonoids inhibit macrophage-mediated oxidation of LDL and attenuate atherogenesis. Atherosclerosis 137, S45-S50.

9. Bagchi D, Bagchi M, Stohs SJ, et al. (2000) Free radicals and grape seed proanthocyanidin extract: importance in human health and disease prevention. Toxicology 148, 187-197. 
10. Bagchi D, Garg A, Krohn RL, et al. (1997) Oxygen free radical scavenging abilities of vitamins $\mathrm{C}$ and $\mathrm{E}$, and a grape seed proanthocyanidin extract in vitro. Res Commun Mol Pathol Pharmacol 95, 179-189.

11. Bumrungpert A, Kalpravidh RW, Chuang CC, et al. (2010) Xanthones from mangosteen inhibit inflammation in human macrophages and in human adipocytes exposed to macrophageconditioned media. J Nutr 140, 842-847.

12. Adisakwattana S, Moonrat J, Srichairat S, et al. (2010) Lipidlowering mechanisms of grape seed extract (Vitis vinifera $\mathrm{L}$ ) and its antihyperlipidemic activity. I Med Plant Res 4, 2113-2120.

13. Vigna GB, Costantini F, Aldini G, et al. (2003) Effect of a standardized grape seed extract on low-density lipoprotein susceptibility to oxidation in heavy smokers. Metabolism $\mathbf{5 2}$ 1250-1257.

14. Preuss HG, Wallerstedt D, Talpur N, et al. (2000) Effects of niacin-bound chromium and grape seed proanthocyanidin extract on the lipid profile of hypercholesterolemic subjects: a pilot study. J Med 31, 227-246.

15. Razavi SM, Gholamin S, Eskandari A, et al. (2013) Red grape seed extract improves lipid profiles and decreases oxidized low-density lipoprotein in patients with mild hyperlipidemia. J Med Food 16, 255-258.

16. Kar P, Laight D, Rooprai HK, et al. (2009) Effects of grape seed extract in type 2 diabetic subjects at high cardiovascular risk: a double blind randomized placebo controlled trial examining metabolic markers, vascular tone, inflammation, oxidative stress and insulin sensitivity. Diabet Med 26, 526-531.

17. Hansen AS, Marckmann P, Dragsted LO, et al. (2005) Effect of red wine and red grape extract on blood lipids, haemostatic factors, and other risk factors for cardiovascular disease. Eur J Clin Nutr 59, 449-455.

18. Moher D, Shamseer L, Clarke M, et al. (2015) Preferred reporting items for systematic review and meta-analysis protocols (PRISMA-P) 2015 statement. Syst Rev 4, 1

19. Jadad AR, Moore RA, Carroll D, et al. (1996) Assessing the quality of curcumin in non-alcoholic fatty liver disease: a randomized controlled trial. Drug Research 67, 244-251.

20. Borenstein M, Hedges LV, Higgins JP, et al. (2011) Introduction to Meta-analysis. Hoboken, NJ: John Wiley \& Sons.

21. Hozo SP, Djulbegovic B \& Hozo I (2005) Estimating the mean and variance from the median, range, and the size of a sample. BMC Med Res Methodol 5, 13

22. Fan J \& Gijbels I (1996) Local Polynomial Modelling and Its Applications: Monographs on Statistics and Applied Probability 66. Boca Raton, FL: CRC Press.

23. Green S \& Higgins J (editors) (2008) The Cochrane Handbook for Systematic Reviews of Interventions. Chichester: Wiley.

24. Clifton PM (2004) Effect of grape seed extract and quercetin on cardiovascular and endothelial parameters in high-risk subjects. J Biomed Biotechnol 5, 272-278.

25. Park E, Edirisinghe I, Choy YY, et al. (2016) Effects of grape seed extract beverage on blood pressure and metabolic indices in individuals with pre-hypertension: a randomised, doubleblinded, two-arm, parallel, placebo-controlled trial. Br J Nutr 115, 226-238

26. Yubero N, Sanz-Buenhombre M, Guadarrama A, et al. (2013) LDL cholesterol-lowering effects of grape extract used as a dietary supplement on healthy volunteers. Int J Food Sci Nutr 64, 400-406.

27. Sano A, Uchida R, Saito M, et al. (2007) Beneficial effects of grape seed extract on malondialdehyde-modified LDL. J Nutr Sci Vitaminol (Tokyo) 53, 174-182.

28. Argani H, Ghorbanihaghjo A, Vatankhahan H, et al. (2016) The effect of red grape seed extract on serum paraoxonase activity in patients with mild to moderate hyperlipidemia. Sao Paulo Med J 134, 234-239.

29. Sivaprakasapillai B, Edirisinghe I, Randolph J, et al. (2009) Effect of grape seed extract on blood pressure in subjects with the metabolic syndrome. Metabolism 58, 1743-1746.

30. Brooker S, Martin S, Pearson A, et al. (2006) Double-blind, placebo-controlled, randomised phase II trial of IH636 grape seed proanthocyanidin extract (GSPE) in patients with radiationinduced breast induration. Radiother Oncol 79, 45-51.

31. Edirisinghe I, Randolph J, Cheema M, et al. (2012) Effect of grape seed extract on postprandial oxidative status and metabolic responses in men and women with the metabolic syndrome - randomized, cross-over, placebo-controlled study. Func Foods Health Dis 2, 508-521.

32. Natella F, Belelli F, Gentili V, et al. (2002) Grape seed proanthocyanidins prevent plasma postprandial oxidative stress in humans. J Agric Food Chem 50, 7720-7725.

33. Vinson JA, Proch J \& Bose P (2001) MegaNatural((R)) gold grapeseed extract: in vitro antioxidant and in vivo human supplementation studies. J Med Food 4, 17-26.

34. Yugarani T, Tan BK, Teh M, et al. (1992) Effects of polyphenolic natural products on the lipid profiles of rats fed high fat diets. Lipids 27, 181-186.

35. Wilcox LJ, Borradaile NM, de Dreu LE, et al. (2001) Secretion of hepatocyte apoB is inhibited by the flavonoids, naringenin and hesperetin, via reduced activity and expression of ACAT2 and MTP. J Lipid Res $\mathbf{4 2}, 725-734$.

36. Pal S, Ho N, Santos C, et al. (2003) Red wine polyphenolics increase LDL receptor expression and activity and suppress the secretion of ApoB100 from human HepG2 cells. J Nutr 133, 700-706.

37. Del Rio D, Costa LG, Lean ME, et al. (2010) Polyphenols and health: what compounds are involved? Nutr Metab Cardiovasc Dis 20, 1-6.

38. Galvano F, La Fauci L, Vitaglione P, et al. (2007) Bioavailability, antioxidant and biological properties of the natural free-radical scavengers cyanidin and related glycosides. Ann Ist Super Sanita 43, 382-393.

39. Grassi D, Necozione S, Lippi C, et al. (2005) Cocoa reduces blood pressure and insulin resistance and improves endothelium-dependent vasodilation in hypertensives. Hypertension 46, 398-405.

40. Engler MB, Engler MM, Chen CY, et al. (2004) Mietus-Snyder. Flavonoid-rich dark chocolate improves endothelial function and increases plasma epicatechin concentrations in Healthy adults. Am Coll Nutr 23, 197-204.

41. Tokede OA, Gaziano JM \& Djousse L (2011) Effects of cocoa products/dark chocolate on serum lipids: a meta-analysis. Eur J Clin Nutrn 65, 879-886.

42. Jia L, Liu X, Bai YY, et al. (2010) Short-term effect of cocoa product consumption on lipid profile: a meta-analysis of randomized controlled trials. Am J Clin Nutr $\mathbf{9 2}, 218-225$.

43. Kondo K, Hirano R, Matsumoto A, et al. (1996) Inhibition of LDL oxidation by cocoa. Lancet 348, 1514.

44. Yamashita Y, Okabe M, Natsume M, et al. (2012) Prevention mechanisms of glucose intolerance and obesity by cacao liquor procyanidin extract in high-fat diet-fed C57BL/6 mice. Arch Biochem Biophys 527, 95-104.

45. Ramos-Romero S, Perez-Cano FJ, Ramiro-Puig E, et al. (2012) Cocoa intake attenuates oxidative stress associated with rat adjuvant arthritis. Pharmacol Res 66, 207-12.

46. Sung JH, Lee SJ, Park KH, et al. (2004) Isoflavones inhibit 3-hydroxy-3-methylglutaryl coenzyme a reductase in vitro. Biosci. Biotechnol. Biochem 68, 428-432.

47. Vinson JA, Mandarano MA, Shuta DL, et al. (2002) Beneficial effects of a novel IH636 grape seed proanthocyanidin extract 
and a niacin-bound chromium in a hamster atherosclerosis model. Mol Cell Biochem 240, 99-103.

48. Giribabu N, Eswar Kumar K, Swapna Rekha S, et al. (2015) Vitis vinifera (Muscat variety) seed ethanolic extract preserves activity levels of enzymes and histology of the liver in adult male rats with diabetes. Evid Based Complement Alternat Med 542026.

49. Tebib K, Besançon P \& Rouanet JM (1994) Dietary grape seed tannins affect lipoproteins, lipoprotein lipases and tissue lipids in rats fed hypercholesterolemic diets. J Nutr 124, 2451-2457.

50. Leifert WR \& Abeywardena MY (2008) Cardioprotective actions of grape poly-phenols. Nutr Res 28, 729-737.
51. Espín JC, González-Sarrías A \& Tomás-Barberán FA (2017) The gut microbiota: a key factor in the therapeutic effects of (poly) phenols. Biochem Pharmacol 139, 82-93.

52. Feliciano RP, Mills CE, Istas G, et al. (2017) Absorption, metabolism and excretion of cranberry (poly) phenols in humans: a dose response study and assessment of inter-individual variability. Nutrients 9, 268

53. Brewer MS (2011) Natural antioxidants: sources, compounds, mechanisms of action, and potential applications. Comprehen Rev Food Sci Food Safety 10 221-247.

54. Leifert WR \& Abeywardena MY (2008) Grape seed and red wine polyphenol extracts inhibit cellular cholesterol uptake, cell proliferation, and 5-lipoxygenase activity. Nutr Res 28, 842-850. 